\title{
VARIACIONES ESPACIALES DE LA TEXTURA EN LADERAS DE EXPOSICIÓN TOPOGRÁFICA CONTRASTADA EN EL SEMIÁRIDO ARAGONÉS
}

\author{
J.C. GONZÁLEZ HIDALGO' \\ M.T. ECHEVERRÍA ${ }^{2}$ \\ F. PELLICER ${ }^{2}$
}

\begin{abstract}
RESUMEN. Las variaciones cumbre-base de la textura de los depósitos de ladera en un ambiente semiárido de la Depresión Central del Ebro (NE España) difieren significativamente según la orientación topográfica. En las solanas las arenas gruesas y finas covarian negativamente con la distancia a la divisoria de aguas y positivamente con la pendiente (0.95). Las mismas relaciones se observan en los limos gruesos, pero con signo cambiado. No se observan relaciones significativas entre la pendiente y la distancia a la divisoria de aguas en las subfracciones de limos finos y arcillas. De la misma manera no se detectan relaciones significativas en cualquiera de las subfracciones analizadas en la umbría. Los resultados se discuten en función de los procesos de erosión bídrica superficial.
\end{abstract}

ABSTRACT. Textural sediment variations between top-bottom slope position bave been studied in a semiarid environment (La Violada, Huesca, NE Spain). Differences are observed according slope aspect. In south aspect the covariability between coarse sand and fine sand with distance from divide is positive ( $>0.95$ ), and negative with slope angle (>0.95). The same relationship is found with coarse silt, but with negative value. There are not been observed significant relationships between fine silt and clay subfraction with distance and slope value. In north aspect slope there are not found any relationsbips between sand, sill and clay fraction with distance and slope value. Results are discussed according to erosion processes.

Palabras Clave: Textura; Laderas; Semiárido; Violada (Huesca, España)

Key words: Grain size; Slopes; Semiarid; Violada (Huesca, Spain)

1. D. Ecología, Universidad Alicante, 03080 , Alicante

2. D. Geografía y Ordenación del Territorio, Ł. Zaragoza, 50009, Zaragoza 


\section{J. C. GONZÁLEZ HIDALGO - M. T. ECHEVERRÍA - F. PELLICER}

\section{Introducción}

Tradicionalmente se acepta que en medios semiáridos la arroyada en superficie es el proceso dominante de erosión. Según esta asunción, a lo largo de una ladera el transporte selectivo del sedimento estaría controlado por el flujo de agua en superficie. HJULSTRÖM (1938) demostró que la velocidad crítica de la lámina de agua, necesaria para la puesta en movimiento (entrainment) de una partícula, se incrementaba a partir de un diámetro de la misma inferior a 0.25 $\mathrm{mm}$, coincidiendo el umbral de velocidad (en torno a $0.2 \mathrm{~m} \mathrm{~s}^{-1}$ ) con el paso de flujos del tipo de transición al flujo laminar (véase BRYAN 1976). Bajo tales condiciones el transporte selectivo de sedimentos se advertiría en un aumento de los tamaños del mismo ladera abajo, concentrándose en partículas de diámetro en torno a $0.25 \mathrm{~mm}$.

Pero la erosión del suelo por procesos hídricos superficiales es el resultado de la conjunción de los procesos de salpicadura y arroyada (MEYER y WISCHMEIER 1969), y en muchas ocasiones la escorrentía superficial no fluye en canales, lo que hace realmente difícil aplicar los criterios de selección del material expuestos.

Las investigaciones sobre los procesos de erosión se han desarrollado aceptando que ocurren en situaciones controladas por el transporte. En tales circunstancias, la cantidad de sedimentos movilizados (léase erosión) es función de la producción de escorrentía superficial y pendiente, y se considera que la disgregación por impacto de la gota de lluvia proporciona material de manera indefinida. Esta es la situación típica de los campos de labor, en donde cabría esperar que el transporte del sedimento se realizase de manera cercana a las ideas expuestas más arriba. Sin embargo, otros trabajos han sugerido la importancia geomorfológica de la disgregación por el impacto de la gota de lluvia, el proceso de salpicadura, y su interacción con la arroyada en el proceso denominado rainwash, que definen un control de la erosión por disgregración, y suele corresponderse con zonas con vegetación espontánea (MORGAN 1986 a; van ASCH 1983; BAUER 1991; GOVERS 1991, etc, aunque también hay opiniones opuestas: PARSON et al 1991).

En el presente trabajo se analiza la variación espacial de las texturas de depósitos de ladera en un ambiente semiárido del interior de España, considerando las variaciones cumbre-base y las relaciones entre fracciones granulométricas y pendiente según la presencia de cubierta vegetal, y se discute, en un marco evolutivo temporal, la importancia relativa de los procesos de erosión hídrica que pueden producir tal granoselección.

\section{2. Área de estudio}

La zona de trabajo se localiza en la margen izquierda de la Depresión del Ebro (Depresión de la Violada, Provincia de Huesca, España, $0^{\circ} 35^{\prime} \mathrm{W}, 24^{\circ} 05^{\prime} \mathrm{N}$ ). 
Litológicamente predominan margas y yesos del terciario sobre los que se configura un relieve alomado de alturas en torno a los $450 \mathrm{~m}$ s.n.m. Climáticamente predominan las condiciones semiáridas; las precipitaciones anuales medias oscilan entre $450-500 \mathrm{~mm}$, están sometidas a grandes irregularidades inter e intra anuales, y presentan dos máximos estacionales de primavera y otoño. No son raros los aguaceros con elevadas intensidades. Los valores de ETP (Estanque A) indican una fuerte demanda ( $>1400 \mathrm{~mm}$ año) que es producto de las elevadas temperaturas sobre todo estivales (media anual $1^{\circ} \mathrm{C}$, Julio y Agosto $>21^{\circ} \mathrm{C}$ ); como consecuencia la cubierta vegetal está formada por matorral xerófilo compuesto de Rosmarinus officinalis, Ononis tridentata, Tymus vulgaris, etc, desarrollada sobre suelos delgados tipo Inceptisol.

En todo el sector es notable la presencia de interfluvios con dirección W-E, y el desarrollo de laderas con exposiciones orientadas al norte y al sur. En estos espacios también es característica común la asimetría de cubrimiento vegetal siendo las formaciones de umbría mucho más densas que las localizadas en solana.

La documentación general sobre la zona es muy abundante, pudiéndose consultar las características edáficas en TORRES (1983), los aspectos geomorfológicos en PELLICER y GONZÁLEZ HIDALGO (1987), GONZÁLEZ HIDALGO (1988 y 1992), los climáticos en GONZÁlEZ HIDALGO (1988, 1989), los botánicos en SERRANO et al (1991).

\section{Materiales y métodos}

Se realizaron tres muestreos cumbre-base en dos laderas de exposición topográfica contrastada norte y sur. La toma de muestras finalizó allí donde desaparecía la vegetación natural y comenzaban los cultivos para evitar los efectos del laboreo. Cada 5 metros se tomaron dos muestras del depósito superficial y se construyeron los perfiles topográficos con ayuda de un pantómetro de $1 \mathrm{~m}$.

La fracción fina $(<2 \mathrm{~mm}$ ) se obtuvo por tamizado en seco tras desagregación manual y limpieza de restos orgánicos y ráces. Por su elevado contenido en yeso, su tratamiento se realizó siguiendo la primera fase del método expuesto por VIELLEIFON (1979) a fin de evitar la floculación. Las subfracciones de arenas gruesas $(2 \mathrm{~mm}-0.2 \mathrm{~mm})$ y finas $(0.2 \mathrm{~mm}-0.05 \mathrm{~mm})$ se obtuvieron por tamizado en todas las muestras. La variación del valor promedio en cada punto de muestreo se redujo mediante nuevos análisis donde fue necesario. En dos muestreos cumbre-base se analizaron las subfracciones de limos y arcillas, desarrollándose el protocolo en su totalidad.

En cada perfil topográfico se evaluó el valor de pendiente que correspondía a los puntos de muestreo, aceptando el promedio de cinco medidas del pantómetro (dos superiores, dos inferiores y la intermedia). Se construyeron los his- 
togramas de frecuencias de cada ladera y se compararon mediante la prueba de Kolmogoroff-Smirnof.

Los valores de las texturas se compararon entre laderas y con la distancia a la divisoria mediante análisis de varianza. La relación con la distancia y pendiente se obtuvo mediante análisis de correlación simple. Asimismo se evaluaron las posibles autocorrelaciones en cada muestreo como indicación de la existencia de granoselección.

\section{Resultados}

En la zona de estudio los perfiles topográficos representativos de laderas de orientación norte y sur difieren morfológicamente. En ladera norte el perfil tipo se inicia en un tramo convexo en divisoria, seguido de un segmento rectilíneo, para dar paso a una concavidad basal. En solana, la convexidad de cumbre es mínima; existe un pequeño escarpe, y el tramo basal cóncavo se prolonga (véase Figura 1). Los análisis realizados en las distribuciones de frecuencias de los valores de pendiente (test de Kolmogoroff-Smirnof) indican diferencias significativas entre laderas (valor Dmax $0.143, \mathrm{p}<0.01$ ), con valores modales superiores en la umbría.

Las subfracciones de arenas gruesas y finas difieren entre laderas $(p<0.05$, Tabla 1). El valor promedio es de $12.54 \%$ y $29.21 \%$ respectivamente para arenas gruesas y finas de la umbría; en solana los promedios son de $9.01 \%$ y $17.78 \%$ respectivamente. En ambos casos el contenido de las subfracciones es superior en ladera norte que en solana ( $\mathrm{p}<0.05$, prueba de Scheffe).

Tabla 1. Análisis de Varianza de las Arenas Gruesas y Finas entre laderas

\begin{tabular}{|c|c|c|c|c|}
\hline & $\mathrm{SC}$ & $\mathrm{gl}$ & $\mathrm{F}$ & $\mathrm{p}$ \\
\hline Ladera" $^{*}$ & 222.09 & 1 & 16.4 & 0.0001 \\
Error & 1005.30 & 72 & & \\
Total & 1234.40 & 73 & & \\
Ladera* $^{*}$ & 2403.34 & 1 & 36.17 & 0.000 \\
Error & 4782.88 & 72 & & \\
Total & 7186.23 & 73 & & \\
\hline
\end{tabular}

Los asteriscos se refieren a las subfracciones de arenas gruesas $\left({ }^{*}\right)$ y arenas finas $\left({ }^{* *}\right)$. SC, suma de cuadrados; gl, grados de libertad; F, estadístico; p, probabilidad. 


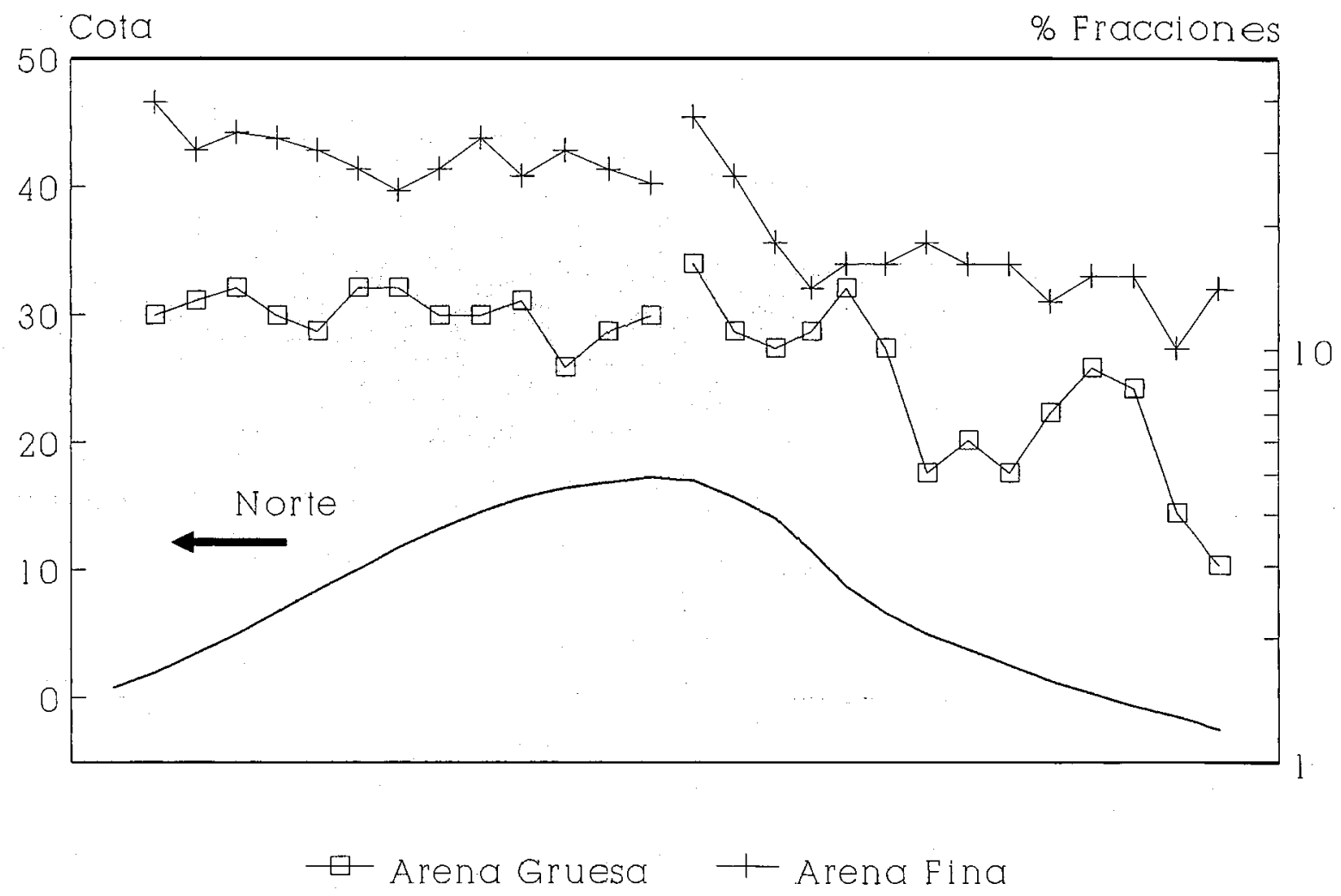

Figura 1. Relaciones entre arenas gruesas (2-0.2 mm), arenas totales $(2-0.05 \mathrm{~mm})$ y pendiente (grados). Ladera sur. En las arenas gruesas los muestros de cumbre se señalan de manera diferenciada.

Las relaciones entre ambas subfracciones con la distancia a la divisoria y el valor de pendiente son distintas según la ladera. En la Tabla 2 se muestran los valores del coeficiente de correlación $\mathrm{r}$ de dichas relaciones.

Tabla 2. Covariación subfracciones de arena (\%) con la distancia a la divisoria (m), y los valores de pendiente (grados)

\begin{tabular}{|c|c|c|c|c|}
\hline Subfracción & N Distancia & N Pendiente & S Distancia & S Pendiente \\
\hline Arena Gruesa & 0.20 & -0.03 & $-0.64^{*}$ & -0.01 \\
Arena Fina & 0.30 & -0.04 & $-0.53^{*}$ & $-0.49^{*}$ \\
Arena Total & $0.41^{*}$ & -0.06 & $-0.67^{*}$ & $-0.37^{*}$ \\
\hline
\end{tabular}

Valores del coeficiente de correlación $r$. Las relaciones significativas (nivel 0.95) se señalan mediante *. Lógicamente la relación limo-arcilla ofrece los mismos valores que la de arenas totales con signo cambiado. 
En ladera norte las arenas gruesas no varían significativamente con la distancia, mientras desciende significativamente su proporción en la ladera sur ( $\mathrm{r}$ 0.64). El porcentaje de arenas finas se incrementa ligeramente con la distancia a la divisoria en la ladera norte $(\mathrm{p}<0.1)$, mientras en la ladera sur la relación es significativamente de signo opuesto (-0.53, Figura 1$)$. Sólamente existen relaciones significativas con la pendiente en la ladera sur con las arenas finas y el total de arenas ( $r-0.49$ y -0.37 respectivamente).

En la ladera sur, la eliminación de las muestras tomadas en interfluvio y los muestreos de cumbre, modifica las relaciones. En la Figura 2 se observa la relación de las arenas totales y las arenas gruesas con la pendiente. Los muestreos de cumbre se señalan de manera diferenciada. Las arenas gruesas mantienen la anterior relación negativa con la distancia $(r-0.67)$, pero presentan una covariación positiva con la pendiente (r 0.69 , significación 0.95 ); las arenas finas dejan de covariar en ambos casos, y en arenas totales desciende el valor de covariación con la distancia ( $r 0.48$ ), pero aumenta con la pendiente y además con signo positivo ( 10.46 ). Las funciones que relacionan la fracción total de arenas y las arenas gruesas con la pendiente (Figura 2) son:

$$
\begin{aligned}
& \text { Arena Gruesa }=0.38 * \mathrm{P}+0.85 \mathrm{R}^{2} 0.48, \mathrm{n} 24 \\
& \text { Arena Total }=0.53 * \mathrm{P}+14.78 \mathrm{R}^{2} 0.21, \mathrm{n} 24
\end{aligned}
$$

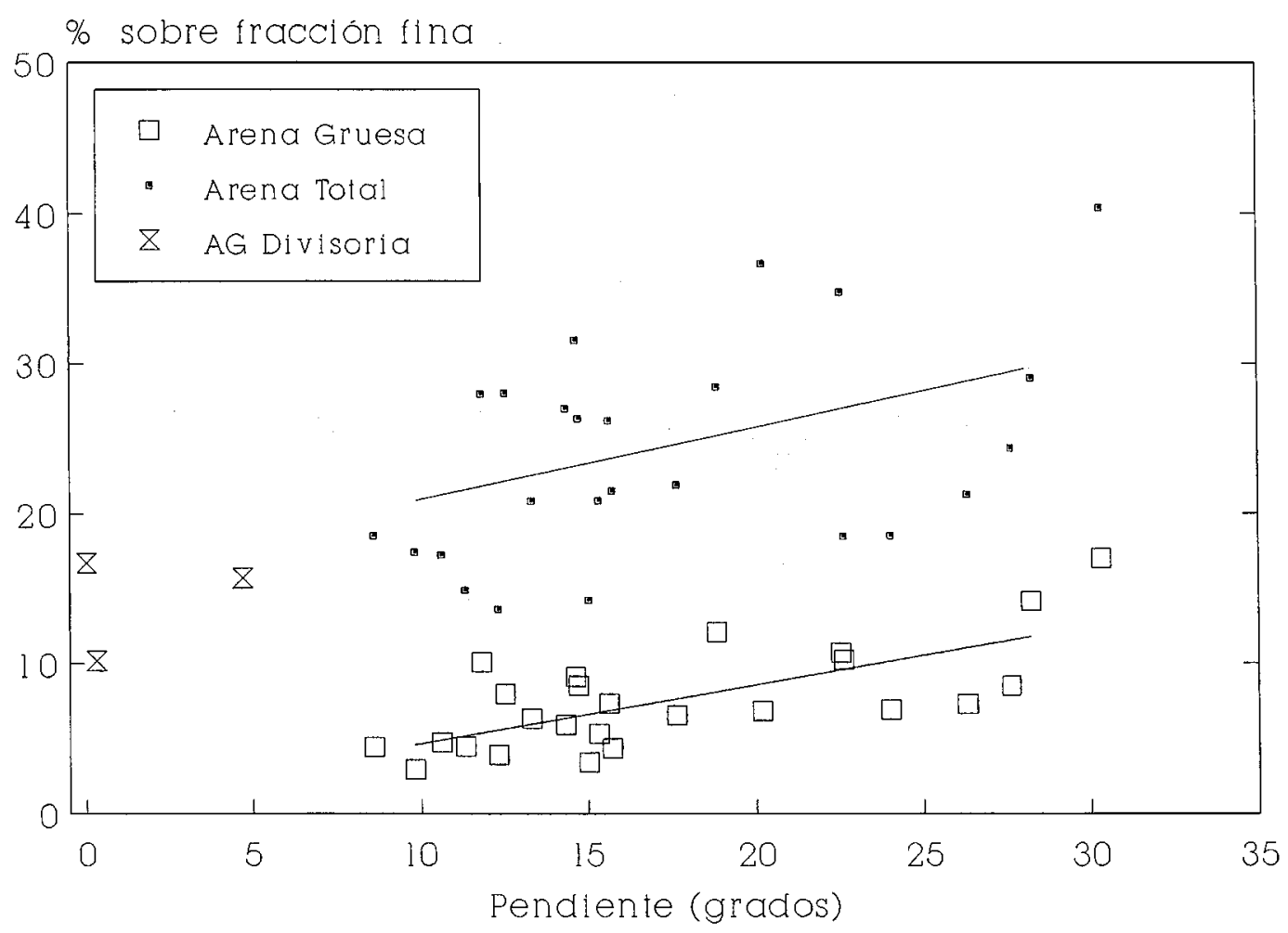

Figura 2. Distribución de arenas gruesas $(2-0.2 \mathrm{~mm})$ y arenas finas $(0.2-0.05 \mathrm{~mm})$ a lo largo de perfiles tipo de ladera con orientación norte (izquierda) y sur (derecha) 
Para comprobar la previsible granoselección de los depósitos se ha efectuado un test de autocorrelación empleando el valor promedio de cada subfracción con la distancia a la divisoria. En la ladera norte los resultados no indican relación significativa; en la ladera sur las dos subfracciones se muestran autocorrelacionadas significativamente (Tabla 3).

Tabla 3. Valores r Test Autocorrelación. Datos promedio de ladera.

\begin{tabular}{|l|c|c|}
\hline & Norte & Sur \\
\hline & & \\
Arena Gruesa & 0.03 & $0.71 *$ \\
Arena Fina & 0.35 & $0.81 *$ \\
Arena Total & 0.49 & $0.83 *$ \\
\hline
\end{tabular}

(") relaciones significativas $(0.95)$. Intervalo de muestreo 1 a partir de cumbre $(5 \mathrm{~m})$.

Los resultados del análisis de las subfracciones de limos y arcillas en un muestreo por ladera permiten definir finalmente los tipos de texturas. En la ladera norte la textura es de tipo franco, predominando en ladera sur el tipo francolimoso (USDA 1975). A pesar de la variación observada en las arenas finas en la umbria, y en las arenas y limos gruesos en solana, no se aprecian variaciones entre cumbre y base.

En ladera norte no existe covariación en alguna de las subfracciones de limos y arcillas con la distancia y la pendiente. En la ladera sur la relación es positiva en los limos gruesos y limos totales con la distancia (r 0.70 y 0.60 , significación $95 \%$ ), pero no lo es con los limos finos y arcillas (Figura 3); la eliminación de muestras de cumbre no supone variar los resultados. Con la pendiente únicamente los limos gruesos covarían significativamente y de manera negativa tras eliminar los muestreos de cumbre ( $\mathrm{r}-0.53$, significación 0.95, Figura 4).

\section{Discusión}

Las variaciones observadas en las texturas de los depósitos del área de Violada indican diferencias significativas entre laderas de orientación topográfica contrastada, y un patrón intraladera definido por la homogeneidad de las umbrías y la heterogeneidad de las solanas. La heterogeneidad en las solanas se manifiesta por el descenso de las subfracciones de arenas y el incremento de limos gruesos hacia la base y por el signo opuesto en su relación con la pendiente. 


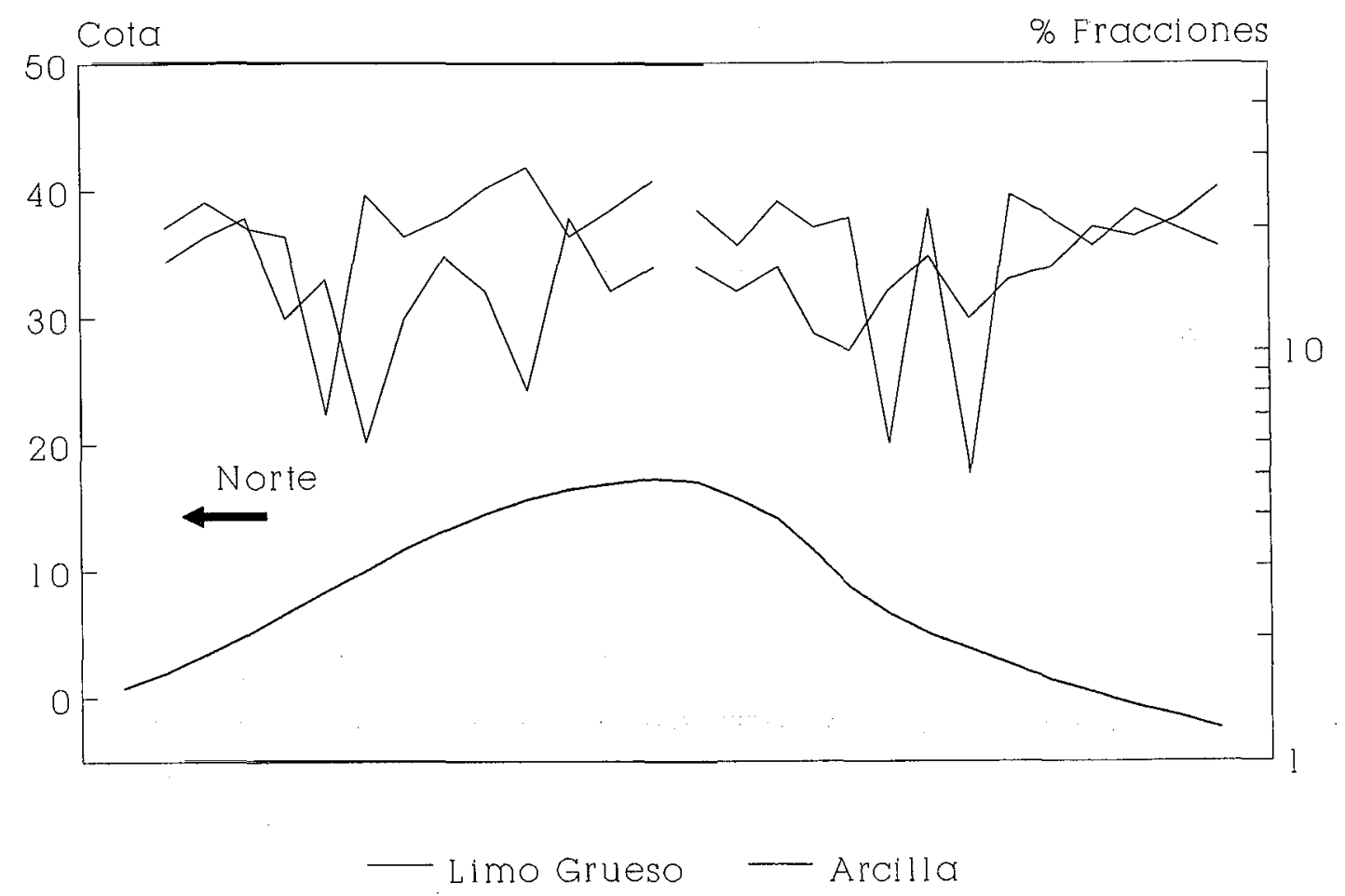

Figura 3. Relación entre limos gruesos (0.05 - $0.02 \mathrm{~mm}$ ) y pendiente (grados). Ladera sur. Se señalan los muestreos de cumbre de manera diferenciada.

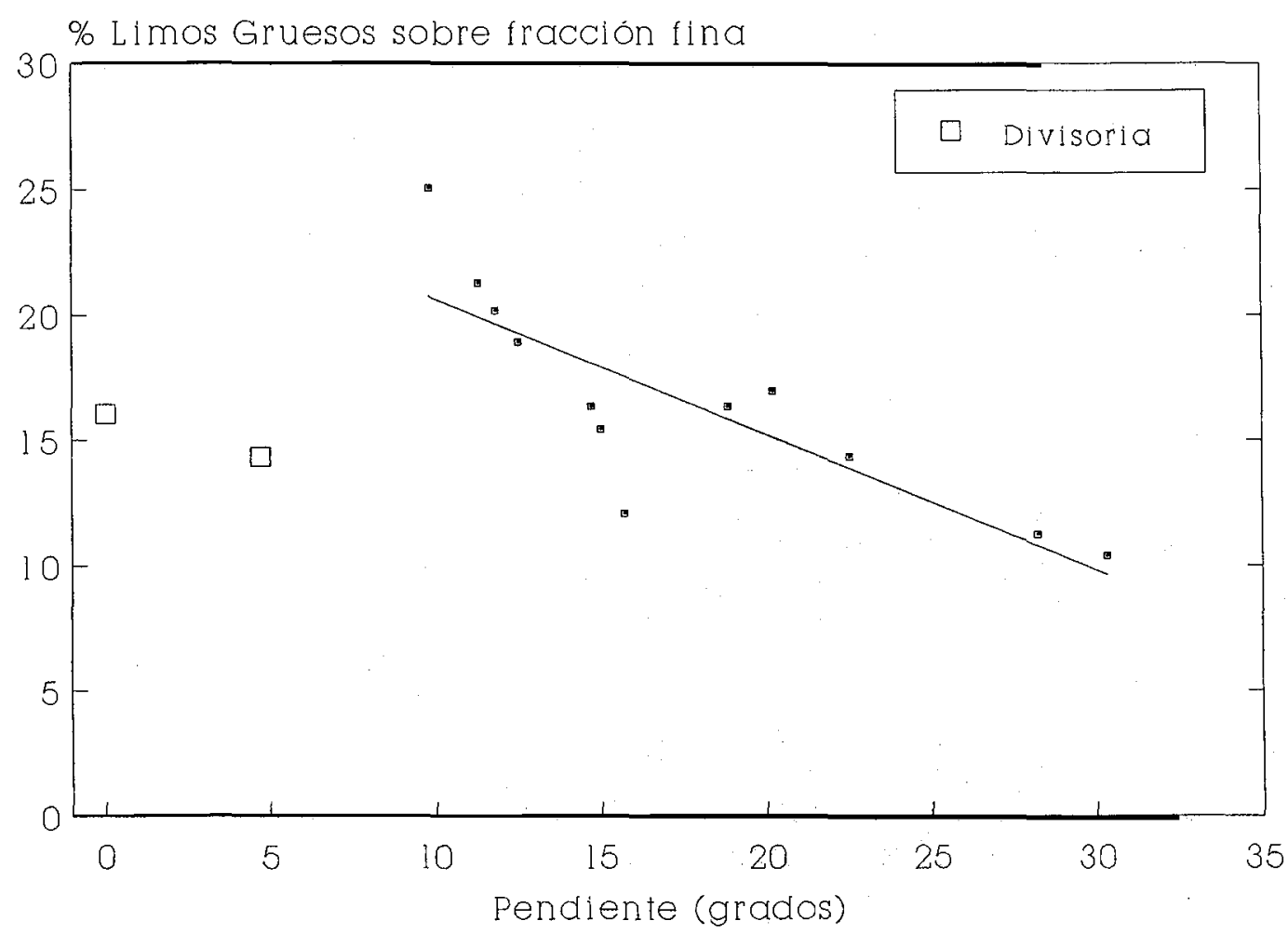

Figura 4. Distribución de limos gruesos $(0.05-0.02 \mathrm{~mm})$ y arcillas $(<0.002 \mathrm{~mm})$ a lo largo de perfiles tipo de ladera con orientación norte (izquierda) y sur (derecha). 
La situación descrita plantea dos cuestiones relacionadas, en primer lugar, con los mecanismos productores de la selección del sedimento y su proyección espacial, y, en segundo lugar, las relaciones encontradas con el valor de la pendiente en las arenas y limos gruesos. Queda pendiente, por no ser propósito de este trabajo, indagar las causas que en origen producen diferentes texturas en laderas de orientación norte y sur. No obstante, se quisiera señalar que en presencia de un mismo substrato, éstas pueden originarse por diferencias dadas en los procesos de meteorización o en los procesos de evolución (transporte y redeposición). Las diferencias en los procesos de meteorización deben considerar que los contenidos de las subfracciones más finas de en solanas (indicadores de mayor meteorización según SMALL 1972) están en aparente contradiccion con la escasa presencia de agua en el suelo en estas laderas (GONZÁLEZ HIDALGO 1992), por lo cual los mecanismos de meteorización física pudieran tener una incidencia no sospechada.

\section{Diferencias cumbre-base entre solana y umbría}

En ambientes como el estudiado (condiciones semiáridas) la erosión del suelo está controlada por procesos superficiales de naturaleza hídrica. En tal situación la granoselección observada en las laderas de estudio debe evaluarse en función de las condiciones de superficie y sus efectos sobre los mecanismos de disgregación y transporte.

En umbría, la presencia de vegetación densa parece ser la causa de la ausencia generalizada de procesos erosivos, y más concretamente de la salpicadura, proceso al que se reconocen escasos efectos bajo cubierta vegetal (OSBORN, 1954; HUDSON 1957; LUK, 1977; BOLLINE, 1980; van ASCH 1983; WIERSUM 1985; MORGAN 1986 a; MORGAN 1986 b). En efecto, el control que ejerce la vegetación sobre el impacto de la gota de lluvia, la escasa disgregación del material de superficie, y en consecuencia el escaso sedimento salpicado, son razones que explican los bajos valores de sedimento producido en estas condiciones y verificados en parcelas experimentales (GONZÁLEZ HIDALGO et al 1992). La homogeneidad cumbre-base de las diferentes fracciones analizadas manifiesta una ausencia de transporte selectivo, que puede explicarse tanto por la escasa incidencia de la salpicadura, como por el escaso poder de disgregación de la arroyada (BOLLINE 1980; MORGAN 1986 a), así como al escaso transporte que ésta realiza, aunque ocasionalmente se hayan producido altos volúmenes de escorrentía (GONZÁLEZ HIDALGO 1992).

En ladera sur la situación es diferente. La vegetación presenta un cubrimiento medio del 50-30\%, y las zonas desnudas abundan. Tanto el impacto de la gota (disgregación), como la salpicadura pueden preparar un sedimento que sea 
arrastrado finalmente por el proceso conjugado de salpicadura y arroyada, el denominado rainwash (MORGAN 1978; van ASCH 1983; MORGAN 1986 a; LUK 1983 etc).

El carácter selectivo de ambos procesos es bien conocido y diversos autores coinciden en que el tamaño del material movilizado se corresponde al de la arena fina, entre 0.2 y $0.05 \mathrm{~mm}$ (POESEN y SAVAT 1980; LANG et al 1984; MORGAN 1986 a; MORGAN 1986 b; POESEN 1986; BAUER 1990; GOVERS 1991; PARSON et al 1991; MARTZ 1992), si bien se compone predominantemente de partículas primarias en el caso de suelos poco agregados y de microagregados en el caso inverso (EGASHIRA y NAKAI 1987; RHOTON y MEYER 1987; MEYER et al 1992). Esta situación se ha podido reconocer en el análisis de las texturas de sedimentos recogidos en parcelas de erosión, en donde se ha observado que la fracción predominantemente movilizada es siempre la arcillo-limosa, con una mayor proporción en las precipitaciones de menor volumen (GONZÁLEZ HIDALGO 1992).

En lógica consecuencia, la selección del sedimento hoy día se realizaría mediante los procesos de salpicadura y rainwash, y afecta preferentemente a la fracción arcillo-limosa que se moviliza en forma de agregados.

\section{Las relaciones con la pendiente}

Una segunda cuestión se refiere a las relaciones de las subfracciones analizadas con la pendiente, y su interpretación en el marco de los procesos erosivos y de evolución del modelado.

Desde un punto de vista morfológico, YOUNG (1972) ha interpretado las distribuciones de los valores de pendiente de los histogramas de frecuencias de una ladera de dos maneras: aceptando que el incremento de desnivel favorece el aumento de los procesos erosivos, y el caso inverso, es decir que el incremento de la pendiente no se corresponde con un aumento, sino con un descenso de la erosión. En el primer caso la situación correspondería a laderas-formas jóvenes, y en el segundo a laderas-formas heredadas.

Tradicionalmente se acepta que el incremento de la pendiente incrementa el arrastre de sedimentos por escorrentía (véase revisión en CARSON y KIRKBY 1972), aunque las condiciones superficiales introducen variaciones en el mismo. BAND (1985) y MATHIER et al (1989) han encontrado exponentes de pendiente $<1$ bien por la selección previa del material o por las variaciones inducidas en la infiltración por el flujo subsuperficial, mientras en ocasiones se ha señalado que la cubierta vegetal también puede alterar los citados exponentes (véase

MORGAN 1986 b p. 59 y ss). Por lo que respecta a la salpicadura, los factores que controlan su capacidad de transporte no son tan claros. MORGAN (1978) y 
POESEN (1986) opinan que la pendiente favorece el transporte positivamente, pero LUK (1977) no ha encontrado dicha relación.

La divergencia que muestran los autores sobre los efectos de la pendiente en los procesos erosivos, particularmente la salpicadura y la arroyada, parece concordar, al menos, en que la pendiente incrementa su efecto hasta un umbral cifrado para la salpicadura en torno a $10^{\circ}$ (LUK 1977; quizá 30 : THORNES 1980), y en la arroyada superficial en torno a $12^{\circ}$ (ABRAHAMS et al 1988), que coincide con el intervalo $10-12^{\circ}$ indicado por SCHUMM et al (1972) y al que se aproxima el valor del intercepto de la función entre arenas gruesas y pendiente mostrado en este trabajo.

En general los trabajos que relacionan granulometría y pendiente se han realizado en fracciones más gruesas a las analizadas en el presente estudio (COOKE y REEVE 1972; KIRKBY y KIRKBY 1974; ABRAHAMS et al 1985 y 1988; PARSON y ABRAHAMS 1986), aunque las relaciones positivas encontradas en Violada, y sus implicaciones geomorfológicas, ya han sido constatadas anteriormente en medios semiáridos (YAIR y KLEIN 1973).

En nuestro caso, las relaciones positivas con la pendiente afectan a la subfracción de arenas gruesas (salvo la divisoria) y a las arenas finas (todo el perfil), y junto a las relaciones observadas en los limos gruesos no se pueden explicar salvo que se acepte el carácter selectivo y predominante de la salpicadura, su control gemorfológico en las laderas estudiadas, y la variación de los efectos de la pendiente sobre los procesos de erosión en función de la cubierta vegetal.

Ya hemos comentado que en las laderas de trabajo se observa que el sedimento se compone mayoritariamente de arcillas y limos en las precipitaciones menores, y que estos sedimentos previsiblemente se transportan en forma de agregados de tamaños de arena fina. Dada la prevalencia de este tipo de eventos pluviométricos en la zona, las consecuencias geomorfológicas inmediatas son: (1) la movilización preferente de los elementos más finos ladera abajo, (2) la diferente actuación de los procesos erosivos entre laderas acorde el grado de protección del suelo, y (3) la modificación del efecto de la pendiente sobre los mencionados procesos debido a la cubierta vegetal.

Frente a las laderas norte, donde la vegetación prácticamente anula la incidencia de la salpicadura y arroyada, las solanas se muestran como espacios de mayor riqueza gemorfológica. En las áreas desnudas de los interfluvios la salpicadura parece ser el proceso dominante y la incidencia de la arroyada escasa. Esta explicación permite entender la presencia de altos valores de arenas gruesas con bajos valores de pendiente (Figura 1 y 2), e incluso una situación semejante con la fracción gruesa $>2 \mathrm{~mm}$ (no mostrada en este trabajo). Ladera abajo, la interacción salpicadura y flujo, en ausencia de vegetación, evacuaría poco a poco las subfracciones más finas en función del incremento de pendiente, par- 


\section{J. C. GONZÁLEZ HIDALGO - M. T. ECHEVERRÍA - F. PEILICER}

ticularmente los limos gruesos en forma de agregados, de manera que la proporción de arcillas y limos finos se mantendría semejante a lo largo del perfil (Figura 3). Por ello, a lo largo de estas laderas los puntos con mayor pendiente quedarían desprovistos de la subfracción de limos gruesos, y se establecería una relación positiva con las subfracciones de las arenas.

La cuestión relativa a la proyección temporal de esta situación no se puede responder de manera definitiva en el estado actual de conocimientos, aunque algunos datos permiten apuntar un diacronismo geomorfológico entre laderas.

En efecto, la ausencia de variaciones en la textura de los depósitos, y los bajos valores de erosión actual pese a unos valores de pendiente superiores, parecen sugerir que las umbrías hoy día están sometidas a condiciones morfogenéticas heredadas que se podrían atribuir al mantenimiento de la cubierta vegetal por las diferentes condiciones topoclimáticas, y sobre todo a las diferentes condiciones de humedad en el suelo. Por su parte, las solanas se presentan como espacios en los que la evolución se realiza más acorde las condiciones del presente. La ausencia de vegetación permite actuar a los procesos de evolución del modelado, que en funcion de la distribución de frecuencias de eventos de lluvia parecen estar dominados por la salpicadura, la cual es el mecanismos responsable preferente de la movilización selectiva del material.

Como quiera que la articulación de los depósitos no puede responder a un inmediato presente, la extrapolación de las condiciones actuales debe llevarnos a la última crisis climática reconocida en el valle del Ebro (Pequeña Edad de Hielo, GUTIÉRREZ y PEÑA 1989), fecha en la que debe datarse, relativamente al menos, el inicio de la situación actual, sin duda alguna relacionada con la diferencia de cubrimientos vegetales entre laderas de orientación contrastada.

En dichos espacios, la interacción entre la dinámica de la vegetación, exposición topográfica y procesos geomorfológicos parece ser la clave de interpretación.

\section{Conclusiones}

Las diferencias texturales observadas entre cumbre y base en el área de Violada se atribuyen a la interacción entre vegetación y procesos de erosión hídrica superficial. En ladera norte no se observan procesos granoselectivos, circunstancia que se atribuye principalmente a la presencia de vegetación densa y su efecto protector de la energía cinética de la lluvia, pese a que la pendiente tenga valores elevados. En las solanas la ausencia de protección del suelo permite atribuir un papel dominante en la evolución geomorfológica actual al mecanismo de salpicadura y su combinación con la arroyada, pese a tener valores de pendiente inferiores a las umbrías. 
En las circunstancias descritas, el desalojo de las subfracciones más finas (previsiblemente en forma de agregados y preferentemente de limos gruesos) provoca un aumento de las subfracciones de arenas en los sectores de mayor pendiente de las solanas. En estas laderas se detecta una división espacial entre las áreas de cumbre y resto del perfil que disloca la evolución general de la ladera e individualiza sectores claramente diferenciados de cumbre, escarpe y concavidad basal.

La proyección temporal de la situación descrita sugiere una hipótesis de trabajo en la que las laderas de orientación norte, cuando tienen vegetación densa, son espacios relictos en los que se han mantenido condiciones de evolución geomorfológica pasadas al amparo de la protección vegetal y condiciones topoclimáticas, mientras las solanas evolucionan de acuerdo con las condiciones del presente.

\section{Agradecimientos}

Los autores expresan su más sincero agradecimiento al Laboratorio de CC TT HH GG de la Universidad de Zaragoza por la financiación de la instalación de campo y las facilidades otorgadas en el trabajo de laboratorio. Del mismo modo se agradece a la Diputación General de Aragón la ayuda otorgada mediante una Beca de Doctorado, que hizo posible el trabajo de campo.

\section{Referencias bibliográficas}

ABRAHAMS, A.; PARSON, A.; HIRSH, P. (1985): Hillslope gradient-particle size relations: evidences for the formation of debris slopes by hydraulic processes in the Mojave Desert. Jour. of Geol., 93: 347-357.

ABRAHAMS, A.; PARSON, A.; LUK, S. (1988): Hydrologic and sediment responses to simulated rainfall on desert hillslopes in Southern Arizona. Catena, 15 : 103-117.

BAND, L. (1985): Field parametrization of an empirical sheetwash transport equation. Catena, 12: 281-290.

BAUER, B. (1990): Soil splash as an important agent of erosion. Geograpbica Polonica, 58: 99-106.

BOLLINE, A. (1980): Splash measurements in the fields. En M. De BOODT and D. GABRIELS (Eds) Assessement of erosion: 441-453

BRYAN, R.B. (1976): Consideration on soil erodibility indices and sheetwash. Catena, 3: 99-111. 
CARSON, M.A. y KIRKBY, M.J. (1972): Hillslope form and processes. Cambridge Univ. Press, Oxford.

COOKE, R. y REEVES, R. (1972): Relations between debris size and the slope of mountain fronts and pediments in the Mojave Desert, California. Zeit. für Geom., 16 (1): 76-82.

EGASHIRA, K. y NAKAI, S. (1987): Size distribution and wet density of sediment eroded under simulated rainfall. Soil Sc. and Plant Nutrition, 33, (3): 347-354.

GONZÁLEZ HIDALGO, J.C. (1988): Geomorfología y procesos morfogenéticos en las márgenes del polígono de riegos de la Violada. Aproximación metodológica. Tesis Msc. ICAMAS, Zaragoza.

GONZÁLEZ HIDALGO, J.C. (1989): Dinámica de ecosistemas: fluctuaciones climáticas de corto plazo. Options Med. Ser. Séminaires, 3: 331-335.

GONZÁLEZ HIDALGO, J.C. (1992): Procesos de erosión bídrica en el semiárido aragonés. Exposición topográfica y cubierta vegetal factores de erosión. Tesis Doctorado, D. Geografia, U, Zaragoza, inédita.

GONZÁlEZ HIDALGO, J.C.; ECHEVERRÍA, M.T.; PELLICER, F.; VALLEJO, R.; BELLOT, J. (1992): Pautas espaciales y temporales de la distribución de la erosión hídrica en el sector central del Valle del Ebro. En F. LÓPEZ BERMÚDEZ et al (Eds) Estudios de Geomorfología en España: 75-82.

GOVERS, G. (1991): Spatial and temporal variations in splash detachment: a field study. Catena Sup. 20: 15-24.

GUTIÉRREZ, M. y PEÑA, J.L. (1989): Upper holocene climatic change and geomorphological processes on slopes and infilled valleys from archeological dating (NE Spain). En European Conference on Landscape Ecological impacts of Climatic Changes, texto manuscrito, $21 \mathrm{p}$.

HUDSON, N.W. (1957): Erosion control research. Progress report on experiments at Henderson Research Station 1953-1956. Rbod. Agric. Journ., 54: 297-323.

HJULSTRöM, F. (1938): Transportation of detritus by moving water. En: P.D.Trask (ed) Recent Marine sediments: 5-31 (traducción del original sueco de fecha 1935).

KIRKBY, A. y KIRKBY, M.J. (1974): Surface water at the semiarid break in slope. Zeit. für Geom. Supp., 21: 151-176.

LANG, K.J.; PRUNTY, L.; SCHOEDER, S.A.; DISRUD, L.A. (1984): Interrill erosion as an index of mined land soil erodibility. Transac. ASAE 27, (1): 99-104.

LUK, S. (1977): Rainfall erosion of some Alberta soils; a laboratory simulated study. Catena, 3: 295-309. 
VARIACIONES ESPACIALES DE LA TEXTURA EN LADERAS DE EXPOSICIÓN CONTRASTADA

LUK, S. (1983): Effect of aggregate size and microtopography on rainwash and rainsplash erosion. Zeit. für Geom., NF 27, 3: 283-295.

MARTZ, L.W. (1992): The variation of soil erodibility with slope position in a cultivated canadian prairie landscape. Earth Surf. Proc. and Landforms, 17: 543-556.

MATHIER, L.; ROY, A.; PARÉ, J. (1989): The effect of slope gradient and length on the parameters of a sediment transport equation for sheetwash. Catena, 16: $545-558$.

MEYER, L.D. y WISCHMEIER, W.H. (1969): Mathematical simulation of the process of soil erosion by running water. Transaction ASAE, 12: 754-758.

MEYER, L.D.; LINE, D.E.; HARMON, W.C. (1992): Size characteristics of sediment from agricultural soil. Journal Soil and Water Conservation, 47, (1): 107-111.

MORGAN, C. (1986 a): The relative significance of splash, rainwash and wash as processes of soil erosion. Zeit. für Geom., 30 (3): 329-337.

MORGAN, R.P.C. (1978): Field studies of rainsplash erosion. Earth Surf. Proc., 3: 295-299.

MORGAN, R.P.C. (1986 b): Soil Erosion and Conservation. Longman, Harlow.

OSBORN, B. (1954): Soil splash by raindrop impact on bare soils. Jour. Soil and Water Cons., 9: 33-38.

PARSON, A. y ABRAHAMS, A. (1986): Gradient-particle size relations on quartz monzonite debris slopes in the Mojave Desert. Jour. of Geol. 95: 423-452.

PARSON, A.; ABRAHAMS, A.D.; LUK, S.H. (1991): Size characteristic of sediment in interrill overland flow on a semiarid hillslope, southenr Arizona. Eartb Surf. Proc. and Landforms, 16: 143-152.

PELLICER, F. y GONZÁLEZ HIDALGO, J.C. (1987): Depósitos del somontano oscense en la depresión de la Violada. Actas $X$ Congreso Nac. Geografía, AGE, T I: 47-56.

POESEN, J. (1986 a): Field measurements of splash erosion to validate a splash transport model. Zeit. für Geom. Supp., 58: 81-91.

POESEN, J. y SAVAT, J. (1980): Particle-size separation during erosion by splash and runoff. En M. De BOODT and D. GABRIELS (Eds) Assessement of Erosion: $427-453$

RHOTON, F.E. y MEYER, L.D. (1987): Sediment size distribution predicted for selected soils. Journal of Soil and Water Conservation, 42, (2): 127-129.

SCHUMM, S.A.; BIRD, J.B.; STARKEL, L. (1970): Report of work group on classification of hillslope. Zeit. für Geom. Supp., 9: 85-87. 
SERRANO, M.M.; GONZÁLEZ HIDALGO, J.C.; AGUINACO, M.T.; BELLOT, J. (1991): Variación espacial de la diversidad en las comunidades de matorral espontáneo dentro de un área antropizada. En F. PINEDA et al, (Eds) Diversidad Biológica: 197-200

SMALL, T.W. (1972): Morphological properties of driftless area soils relative to slope aspect and position. Prof. Geogr., 24 (4): 321-326.

THORNES, J.B. (1980): Erosionl processes of running water and their spatial and temporal controls: a theoretical viewpoint. En M. J. KIRKBY and J. B. THORNES (Eds) Soil Erosion: 129-182

TORRES, M.M. (1983): Balance hidrosalino de un polígono de riego en los llanos de la Violada. Tesis Master, CIHEAN, Zaragoza.

USDA (1975): Agriculture Handbook. USDA Soil Cons. Service, publ. n 436.

VIELLEIFON, J. (1979): Contribution à l'amelioration de l'étude analytique des sols gypseux. Cabiers ORSTON, Ser. Ped., 17 (3): 195-223.

Van ASCH, Th. W. J. (1983): Water erosion on slopes in some land units in a mediterranean area. Catena, 4: 129-140.

WIERSUM, K.F. (1985): Effects of various vegetation layers of an Acacia auriculiformisforest plantation on surface erosion at Java, Indonesia. En S.A. ElSWAIFY et al (Eds) Soil erosion and conservation: $79-89$

YAIR, A. y KLEIN, M. (1973): The influences of surface properties on flow and erosion processes on debris slopes in an arid area. Catena, 1: 1-18.

YOUNG, A. (1972): Slopes. Longman, Edimburgo. 